\title{
Innovative Drone Design for the AHS Micro Air Vehicle Competition
}

\author{
Bobby Tang Dan, Utsav Shah, Mechatronics Engineering \\ Vaughn College of Aeronautics and Technology,NY, USA, bobby.tang@vaughn.edu,utsav.shah@vaughn.edu \\ Mentor: Amir Elzawawy, Ph.D., Hossein Rahemi, Ph.D. \\ Vaughn College of Aeronautics and Technology,NY, amir.elzawawy@vaughn.edu, hossein.rahemi@vaughn.edu
}

\begin{abstract}
The project outlines a project idea of creating two micro aerial vehicles weighing less than 500 grams and less than 450 millimeters in any dimension. The quadcopters designed in this project are two different types of package delivery and pickup drones capable of autonomous and manual control. As a part of the American Helicopter Society International 2017 Micro Air Vehicle Student Challenge, the drones' design follows strict restrictions with the necessity of high performance and endurance. In this project, the drones will be developed to provide a pilot with the ability to control the drone via a radio transmitter and First Person View (FPV) goggle headset. The autonomous drone, however, will be designed to incorporate multiple sensors capable of target detection and obstacle avoidance. Optimal designs, including systematical consideration of the drones' mechanical and electrical performance, have been developed for both autonomous and pilot control modes. Engineering analysis on the stress and strain of the micro air vehicles and various experiments for the comparison of system performances have been conducted through CATIA software and field test approach. The results demonstrate the successfulness of the designed mechanical drone systems.
\end{abstract}

Keywords- Drone, Design, Build, Program, Manual, Autonomous

\section{INTRODUCTION}

\section{A. Competition Overview}

The 2017 Micro Air Vehicle (MAV) Student Challenge takes place at the American Helicopter Society International $73^{\text {rd }}$ Annual Forum [1]. The competition task is to design and build an aerial vehicle that is less than 500 grams (17.6 oz.) in weight, including batteries and less than $450 \mathrm{~mm}$ (17.7 in.) in any dimension. The quad-copter would be required to be able to perform a vertical take-off and landing and electric powered. The aerial vehicle must be able to take off from a home base, hover, fly towards the targets while avoiding an obstacle, find the first delivery target and perform a precise package drop. After delivering the first package, the vehicle must then find the second target, pick up another package, and return to home base avoiding the obstacle. The delivery package in this competition is a sealed, standard envelope containing 25 grams of paper. Slight modifications can be made to the first delivery package without damaging the envelope. The second package has a red ribbon tied around it. The obstacle in this competition is a 3-D structure, consisting of foam board sides and coloured to resemble a skyscraper in
Fort Worth. In dimension, the obstacle is approximately 10 feet tall, 5 feet wide, and 5 feet deep.

The Micro Air Vehicle Student Competition is divided into three separate gates (assessment periods) that must be completed throughout the year. The first two gates of the competition consist of paper and video submissions that were sent to the competition coordinator. After the first two gate submissions have been completed, a team of judges will decide the six finalist teams that will compete at the third gate of the competition. The criteria used to pick the finalist teams include prior experience, aircraft design diversity, proof of vehicle readiness, vehicle weight and size constraints, and plan execution.

\section{B. Gate Submissions}

For the first gate, each participating team is required to submit a completed team information form with a short synopsis about the micro air vehicle that will be used in the competition.

1. The paper submission should include the following information:

- Team description, with key student and faculty members. Includes the major and year of each student member. (1 page).

- The micro air vehicle specification and capabilities (1 page).

- The onboard-system and remote-operation development proposal ( 2 pages).

- The preliminary project schedule and plan to the final demonstration. (1 page).

In the second gate, each participating team is required to submit video evidence of the autonomous or remote controlled micro air vehicle along with a second paper describing the final vehicle.

1. The video should include the following requirements and criteria.

- Measurement of the vehicle dimensions and weight.

- The micro air vehicle achieving stable hover capability.

- The ability of the vehicle to fly a simple version of the mission course.

- Show target recognition capabilities utilizing the onboard systems. 
- A preliminary demonstration and proof of a package drop-off \& pickup capability.

- Teams entering the remotely operated category should clearly show the vehicle being piloted using only onboard systems.

- Teams entering the autonomous category should ensure that the video is recorded while the autonomous mode is active with a real-time groundstation or recorded target-feedback.

- This information should be relevant to the delivery/pickup site recognition and obstacle avoidance.

2. The paper submission should include the following information:

- The final micro air vehicle specification and configuration description. ( 2 pages)

- The final vehicle autonomy and remote operation capabilities ( 2 pages)

- This portion of the paper should include target tracking results.

- The package delivery and pickup system description. (1 page)

- The gaps that will be addressed before the final competition.

The third gate of the competition is the final portion of the competition. It consists of a live demonstration by the six finalist teams chosen. In this part of the competition, teams will be required to complete two tasks. For the first task of the competition is a short poster presentation about their micro air vehicle as well as perform a short free-flight demonstration. The teams will be judged based on a set of design and innovation metrics shown below.

TABLE I.

TASK A DESIGN AND INNOVATION METRICS

\begin{tabular}{|c|c|c|}
\hline Category & Design and Innovation Metrics & $\begin{array}{c}\text { Rating ID } \\
(0-5)\end{array}$ \\
\hline Originality & $\begin{array}{l}\text { Not off-the-shelf, involves innovative } \\
\text { engineering, solved challenging } \\
\text { integration issues, unique capabilities }\end{array}$ & A1 \\
\hline $\begin{array}{c}\text { Flight } \\
\text { Performance }\end{array}$ & $\begin{array}{l}\text { Physical design, stabilization } \\
\text { approach, aerodynamics and flight- } \\
\text { stability, agility }\end{array}$ & A2 \\
\hline $\begin{array}{c}\text { System } \\
\text { Engineering }\end{array}$ & $\begin{array}{l}\text { Sensor-integration, remote pilot and } \\
\text { ground control station capabilities, } \\
\text { antenna, wiring, sensor installation, } \\
\text { craftsmanship } \\
\end{array}$ & A3 \\
\hline Maturity & $\begin{array}{l}\text { Ruggedness, field readiness, } \\
\text { completeness of design, autonomy- } \\
\text { readiness, potential for sensors. }\end{array}$ & A4 \\
\hline Teamwork & $\begin{array}{l}\text { Inter-disciplinary teams, team } \\
\text { "intangibles", leadership shown. }\end{array}$ & A5 \\
\hline
\end{tabular}

The second segment of the third gate is the actual mission flight demonstration and completion of tasks outlined previously in the competition. Each team is given a total of ten minutes to perform the mission tasks. Teams are given the chance to declare a second attempt of the mission field if they believe it is necessary. However, the clock does not stop for any necessary preparations of the second attempt. As a result, all pilot trims, battery changes, and field resets are included within the time limit.

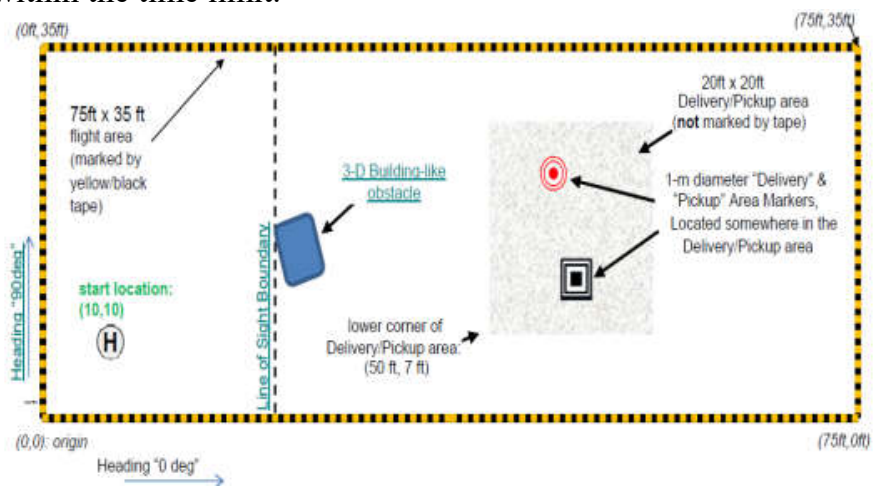

FIGURE I. MISSION TASK LAYOUT

\section{ENGINEERING REQUIREMENTS}

1) Strength and Durability

a. The landing gear pickup/delivery mechanism must be able to withstand 500 grams of force.

b. The main frame of the body must be rigid with minimal flexibility.

c. The quad-copter must withstand the thrust from the motors.

d. The frame must be able to withstand high strain and stress.

2) Vibration Dampening

a. The quad-copter must be able to dampen the motor vibrations to prevent disorientation of the flight controller.

3) Design

a. The quad-copter must be within the vehicle specifications defined by the competition.

i. Weight $<500$ grams (1.1 pound)

ii. Dimension measures $<450$ millimeters ( 17.7 inches) in any direction

b. The design of the quad-copter must be able to contain all the parts necessary for flight on the quad-copter.

c. The various parts of the frame must be designed to allow for easy replacement when damaged or broken.

d. The frame must be designed to make the quad-copter move dexterously. 


\section{4) Programming}

a. The quad-copter for the manual competition must be fine-tuned in P.I.D. control to allow the stable flight and effective regulation.

b. The servos must be programmed to move to the correct orientations for piloted control.

i. The servo for the Mini-FPV (First Person View) camera must be programmed to have three distinct position sets by the auxiliary switches.

ii. The continuous servo for the manual competition drone must be set to stop when the pickup mechanism is fully extended or closed.

c. A power termination switch must be programmed to immediately turn off all motors in the case of an emergency.

\section{SYSTEM DESIGN AND REQUIREMENTS}

\section{A. Manual Mode}

The centre of the quad-copter is the flight controller which regulates the actions and movement of the drone. The flight controller is a circuit board that generates the motor speed based on user commands and the sensory data gathered. The basic flight controller consists of sensors such as gyroscopes and 3-axis accelerometers. More advanced flight controllers also have a barometer, magnetometer, and global positioning system (GPS). On the market today, there are a broad range of flight controllers including the Naze32 for racing drones and the Ardu-pilot Mega (APM) for autonomous drones [7].

Another critical component of the quad-copter is the electronic speed controllers (ESCs). The ESCs control the speed of the motor based on the input signals generated by the flight controller. In general, the ESC consists of a power, ground, and receiver cable on one side and three wires on the other side. The three wires perform the same function and create an alternating current circuit with the brushless motors. Many ESCs on the market today come with a feature called Battery Elimination Circuit (BEC) which supplies power to the radio receiver and the flight controller. The main purpose of the BEC feature is to eliminate the need of a separate power source to power the different components onboard the quadcopter. The key factors to be considered when choosing the correct ESCs are the current rating, voltage rating, and a BEC feature. A vital rule of choosing the correct speed controllers for the motors is to ensure that the current rating is higher than the brushless motor chosen. This is important as the motors could potentially draw more amperage than the stated ratings and cause the electronic speed controllers to burn out.

The quad-copter houses a Flyduino Kiss CompactCTRL 32-bit flight controller. This includes a gyroscope, accelerometer, and barometer. The flight controller also has four built in 16-amp electronic speed controllers (ESCs) and a power distribution board. This eliminates the necessity for an additional power distribution board and four ESCs. In the first building of the manual control quad-copter, a Naze32, power distribution board, and four Arris 20A ESCs weighed a total of 48 grams. As a result, it could be seen that the use of the Flyduino Kiss flight controller eliminated a total of 37 grams of weight.

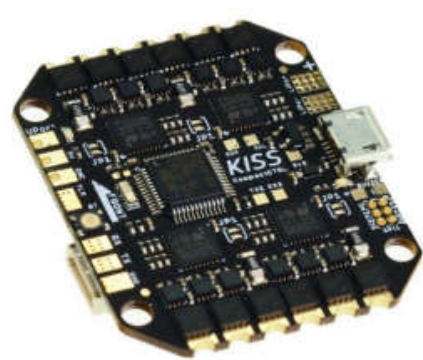

FIGURE II.

FLYDUINO KISS COMPACTCTRL FLIGHT CONTROLLER

The commonly used motors chosen are the direct current brushless electric motors instead of traditional direct current brushed motors [10]. The brushless motor does not use brushes or commutators. Instead, it uses a small circuit that coordinates the energy delivery to the windings. To choose the correct motors for the quad-copter, the motor data sheet is required to calculate the total thrust that the four motors can produce with a specific type of propeller. The motors that have been chosen for this quad-copter are Arris 1806 2300KV brushless motors which are lightweight and provide enough thrust for the quad-copter to lift itself and the package for the assigned task. The total thrust that each motor is 210 grams as seen in the motor data sheet provided in Appendix B. Since the total weight limit of the quad-copter set by the competition is 500 grams, the total thrust is enough for the quad-copter to lift off and maneuver.

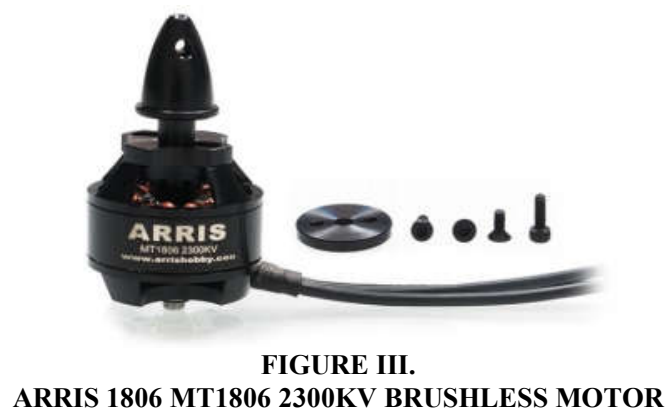

The most commonly used type of battery for drones is the Lithium Polymer (LiPo) battery. In comparison to other batteries, LiPo batteries provide longer flight times and can discharge substantial amounts of power. Furthermore, LiPo batteries are lighter in weight, have higher power capacities and discharge rates. The key factor in selecting the correct battery for the drone is to check the specifications of the brushless motors and electronic speed controllers. It is 
imperative that a higher rated battery is never plugged into a component that is not compatible. A large factor in the weight of the battery is the capacity of the LiPo battery. As the capacity of the battery increases, the weight does so as well. For the competition, there is a ten-minute time limit which means that the drone does not require a large amount of flight time. As a result, the battery chosen for this quad-copter is a Venom Fly 3S 1300mAh LiPo battery.

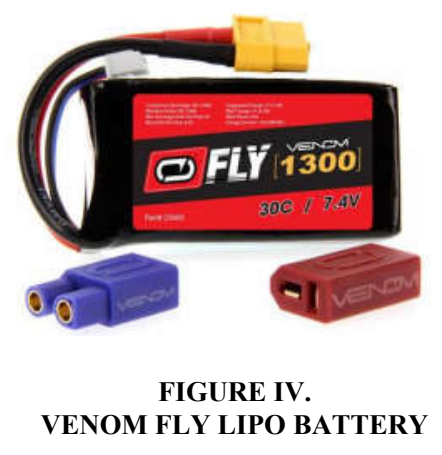

The last component required in building the main body of the drone is the propellers. The two types of propellers available on the market today are plastic and carbon fiber. In choosing the correct propeller for a drone, there are two dimensions that should be considered. These are the size and pitch of the propeller. The size of the propeller dictates the minimal size that the frame of the quad-copter can be to ensure that the propellers will not come in contact with each other when the motors spin. The pitch of the propeller gives information regarding the traveling distance of each revolution of the propeller. A lower pitch propeller generates more torque for lift, while a higher pitch propeller can displace a greater amount of air. However, higher pitched propellers produce less torque and more turbulence. The type of propeller that is used can dictate the efficiency of the motors and the maximum flight time.

Due to the competition requirement that the pilot is not allowed to view the field after passing the line of sight, a FirstPerson View (FPV) camera is required. An FPV camera is a video camera that is mounted on the quad-copter and broadcasts live video to the pilot on the ground as the aircraft flies. This allows pilots to navigate in higher altitudes and to locations where line of sight is not possible. The traditional FPV camera systems consist of a bulky camera, power module, transmitter system and antenna. To minimize the total weight of the quad-copter, the Eyoyo three-in-one FPV camera was chosen for its lightweight and compact design.

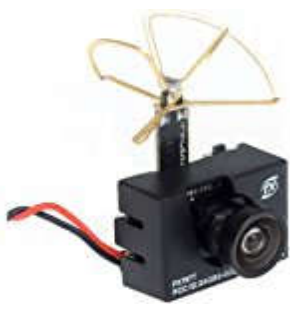

FIGURE $V$. EYOYO MINI FPV CAMERA

A Taranis FrSky Transmitter X9D Radio Transmitter will be used for remote operation of the micro aerial vehicle. The transmitter houses several auxiliary switches which can be programmed to control several functionalities including controlling the servos, arming the drone and several other functions. Onboard the drone is a X6R 16 channel receiver that was bound to the transmitter. This receiver was used due to the number of channels available to control all components of the quad-copter. The total weight of the receiver was measured to be 15 grams.

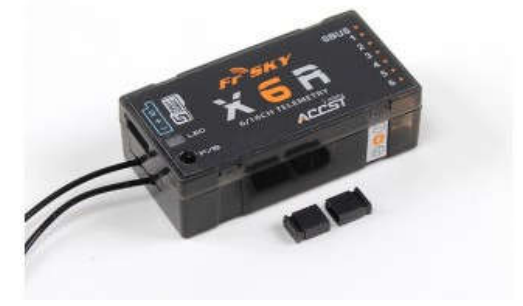

FIGURE VI. FRSKY X6R RADIO RECEIVER

To perform the mission tasks, the different components of the drone are actuated using servos. The FPV camera was attached to a $90^{\circ}$ servo that was programmed to allow the pilot to control the angle of the camera using an auxiliary dial on the FrSky Taranis transmitter. The second servo utilized on the quad-copter is a continuous rotation servo for the pickup/drop-off mechanism. A continuous servo was used instead of a $90^{\circ}$ servo due to the required distance to completely extend or close the mechanism. Like the camera servo, the mechanism servo was programmed to an auxiliary dial on the transmitter.

\section{B. Autonomous Mode}

The flight controller chosen for the autonomous category quad-copter is the Pixhawk 2.0 flight controller. This flight controller differs from the KISS CompactCTRL flight controller in both processing power and autonomous capabilities. The flight controller can connect to multiple sensors such as the Lidar Lite and PX4 Optical Flow. The total weight of the Pixhawk flight controller was measured to be 19 grams. 


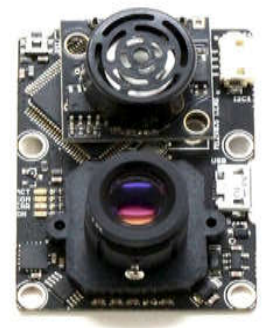

\section{FIGURE VII. PX4 OPTICAL FLOW}

The Lidar-Lite V3 sensor is a laser rangefinder sensor that functions as a proximity sensor for drones, robots, and any other types of unmanned vehicles. The Lidar-Lite 3 is compact, lightweight, and has low power consumption. The sensor allows adjustment between accuracy, operating range, and measurement time with communication via I2C and Pulse Width Modulation (PWM). The weight of this sensor was measured to be a total of 20 grams. In addition to the LidarLite, another sensor that was required for the competition was the PX4 Optical Flow. The PXR Flow smart camera is a specialized downward facing camera module with a 3-axis gyro using the ground texture, visible features, and a separate range finder (Lidar-Lite 3) to determine aircraft ground velocity. The camera was measured to weigh a total of 19 grams. The two sensors work in unison to provide the micro air vehicle with sensory data and help determine its location.

Another essential component of the autonomous control quad-copter is the Odroid XU4 computer board. The Odroid XU4 comes equipped with a Samsung Exynos5422 Cortex A15 $2 \mathrm{GHz}$ processor and 2 Gigabytes of LPDDR3 RAM memory. The purpose of the Odroid is to perform the onboard processing of target recognition and provide commands to the flight controller based on the sensory data gathered. The Odroid XU4 comes also fully equipped with a heat sink and fan cooling system resulting in a total weight of 40 grams. The Raspberry Pi Cam V2 was connected to the Odroid XU4 computer providing live video feed for the target recognition software. The camera was also chosen due to the light weight and compact design requiring less space on the frame to mount. Including the ribbon cable for the camera, the total weight was measured to be 2 grams.

A similarity between the manual controlled quadcopter, the autonomous quad-copter was equipped with four Arris 1806 MT1806 2300KV brushless motors with 5030 Gemfan propellers. Since the Pixhawk flight controller does not have built in electronic speed controllers, the micro air vehicle requires four Arris 20A ESCs weighing a total of 21.2 grams. To supply the motors and quad-copter with power, a 5.5-gram solder contact power distribution board was used to connect the different electrical components of the quad-copter [6].

\section{VEHICLE FRAME DESIGN}

\section{A. Autonomous Mode}

The autonomous control micro air vehicle type chosen was a quad-copter with a cross shaped motor mount design. The quad-copter design was selected due to the stability and manoeuvrability of the micro air vehicle for autonomous flight. The main portion of the frame follows a square pattern consisting of carbon fiber rods and motor mounts printed using Polylactic Acid (PLA) on the Makerbot $5^{\text {th }}$ Generation Replicator [10]. Furthermore, a second elevated plate was designed to house the different components such as the Pixhawk flight controller.

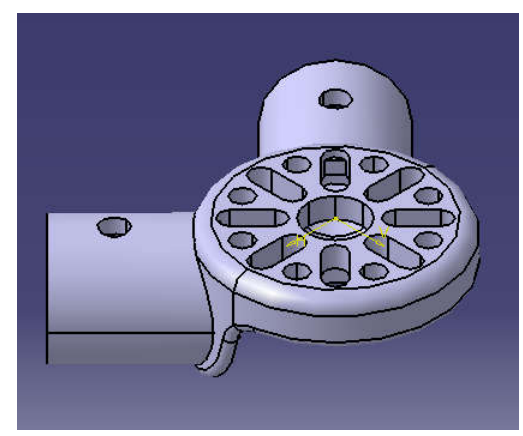

FIGURE VIII.

AUTONOMOUS DRONE MOTOR MOUNT CATIA RENDERING

The overall frame was designed to be several different pieces that would be fully assembled to create the quad-copter frame. The frame consists of four motor mounts that are connected using four 0.315 -inch diameter carbon fiber rods cut at 6.5 inches. The rods were cut at 6.5 inches because it was the smallest size the frame can be while providing enough clearance for the propellers. The carbon fiber rods are wrapped with a vibration dampening mat before being connected to the motor mounts. The purpose of the vibration dampening mat barrier is to prevent the vibration generated by the brushless motors from reaching the onboard sensors and flight controller. The motor mounts were designed with a diameter of $30 \mathrm{~mm}$ and a 2-mm wall thickness. As seen in Figure 11, the motor mount was designed with two carbon fiber rod mounts perpendicular to each other.

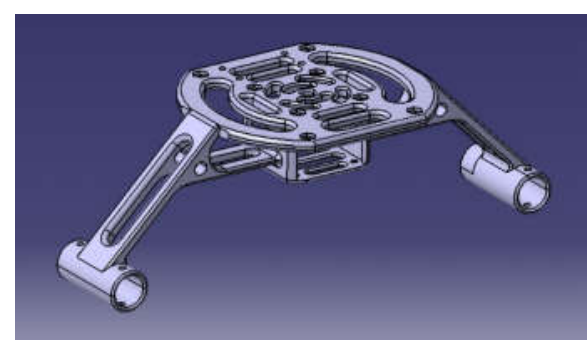

FIGURE IX.

AUTONOMOUS DRONE UPPER MOUNT CATIA RENDERING

$15^{\text {th }}$ LACCEI International Multi-Conference for Engineering, Education, and Technology: "Global Partnerships for Development and Engineering Education”, 19-21 July 2017, Boca Raton FL, United States. 
The central piece of the quad-copter consists of a flat plate with two stands that are angled to attach to the carbon fiber rods between the motor mounts. To prevent the legs of the plate from breaking due to bending force, a center beam was created connecting the legs to the battery compartment as seen below. In the design of the frame, hexagonal pockets were created at specific distances set by the dimensions of the electrical components required to program autonomous flight. The thickness of all parts of the frame was designed with a 3$\mathrm{mm}$ thickness to prevent flexibility.

The different components were printed with a hexagonal pattern at an infill setting of 30\%. The infill setting was 30\% due to multiple tests in the past. The infill setting provides enough strength and durability for flight while remaining relatively light weight. As a result, the hexagonal pattern at $30 \%$ infill is the most optimal setting for printing at 45 degrees on the print bed. In the figure below, the final print and build of the drone frame is shown.

\section{B. Manual Mode}

The micro air vehicle chosen for the competition is a quadcopter with a 3D printed frame. The frame of the quad-copter was designed to be as compact as possible with a cross shaped frame for stability. In designing quad-copters, cross shaped designs are used for stable flight while $\mathrm{H}$ - shaped designs are for high speed racing drones. The drone frame was split into three separate components: the main frame, the pickup/dropoff mechanism, and the landing gear. Separating the drone into three separate components allows for a quick replacement if any part of the drone is broken. The quad-copter is designed to have a dimension of $278 \mathrm{~mm}$. (10.9 in.) in diagonal from propeller to propeller and weight of $300 \mathrm{~g}$ without battery. The manual drone's frame was printed as one solid piece. The reason of designing the drone in this way is due to the characteristics of 3-D printing. A part is less likely to break if it is printed as one solid piece.

The main body of the drone was designed following a cross shaped quad-copter design with a square center. The square center of the quad-copter was designed to be large enough to house the Flyduino KISS CompactCTRL flight controller. The hexagonal pockets seen in figure 12 were created to mount the flight controller using plastic M3 hexagonal standoffs. At the center of the square, a cross shaped pocket was created to mount the Towerpro $9 \mathrm{~g}$ servo to the bottom of the frame. At the bottom of the frame, two slide mounts were created to hold the rack and pinion landing gear mechanism. The rack was dimensioned at an offset of $7.5 \mathrm{~mm}$ from the bottom of the frame to remain level with the gear of the servo. Lastly, the arms of the quad-copter were designed to have two layers with the motor mounted on the upper layer. The purpose of this was to increase the distance the vibration generated by the motors would travel to reach the flight controller. This increased distance aids in dampening the vibration over time.

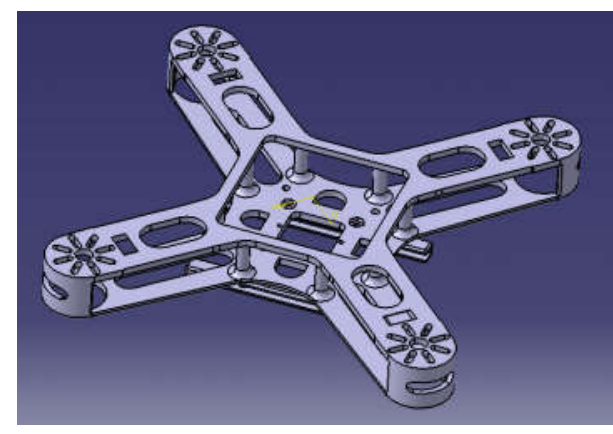

FIGURE $X$.

MANUAL DRONE FRAME CATIA RENDERING

The second portion of the quad-copter is the mechanism for the retrieval and delivery the envelope package. The mechanism is designed to be proportional and symmetrical to the main body of the frame to support and distribute the weight of the micro air vehicle. As a result, the pickup/drop-off mechanism is also utilized as the landing gears for the quadcopter. The mechanism consists of a slide plate, two rack gears, a pinion gear, and two claws.

The different components were printed with a hexagonal pattern at an infill setting of 30\%. The infill setting was 30\% due to multiple tests in the past. The infill setting provides enough strength and durability for flight while remaining relatively light weight. As a result, the hexagonal pattern at $30 \%$ infill is the most optimal setting for printing at 45 degrees on the print bed. The total print time on the Makerbot Replicator and Formlabs Form 2 for all components of the frame was 43 hours. In the figure below, the final print and build of the drone frame is shown.

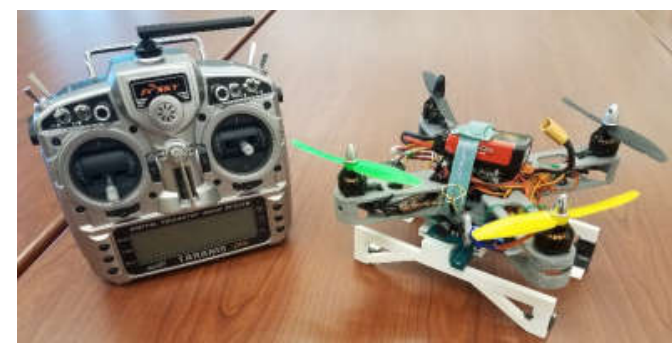

FIGURE XI.

MANUAL DRONE FINAL BUILD 


\section{PACKage Delivery ANd Retrieval System}

\section{A. Manual Mode}

The package delivery and retrieval system consists of a two rack gears and a pinion gear that would be actuated with a continuous rotation servo motor. A plate with two guide rails was created to hold the rack and pinion system. At the end of each rack gear, is a removable claw that can be changed to suit the varied sizes of the guide post used.

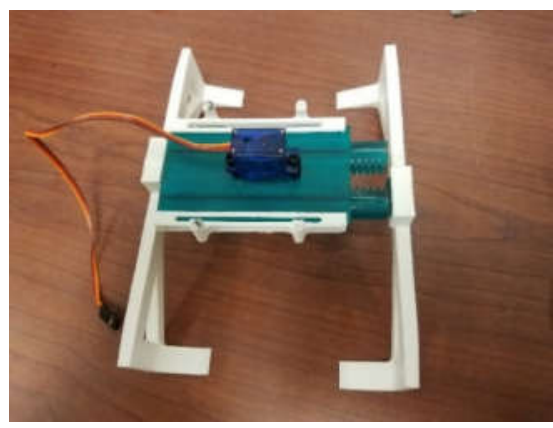

FIGURE XII.

PACKAGE MECHANISM TOP VIEW

The claws are designed at three different dimensions to allow for clearance of the guideposts that will be used and can be detached from the pinion gears. The claws were printed with a 1-inch, 1.5-inch, and 2-inch clearance. The claw system was printed at $30 \%$ infill with a hexagonal pattern. The hexagonal pattern allows for a stronger design necessary to withstand the force of impact when the drone lands. The center plate, rack gears, and pinion gear were printed on the FormLabs 2 using tough resin to withstand continuous testing and use.

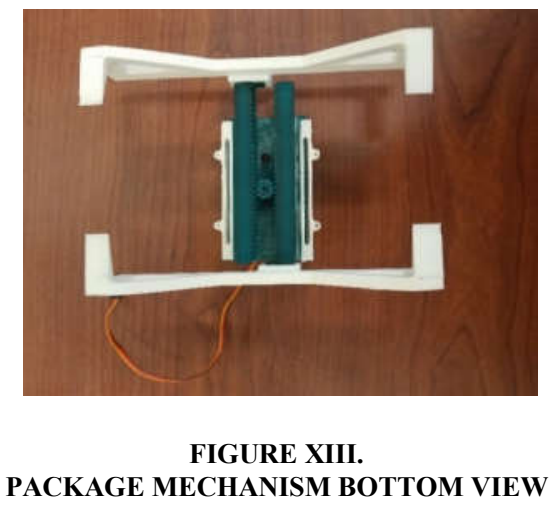

The rack gears of the pickup mechanism were designed to have 33 teeth while the pinion gear has 10 teeth. This creates a 3:1 ratio between the rack and pinion which suffices in providing just enough speed to actuate the mechanism in an efficient manner. In fine tuning of the design, several iterations were done to ensure that the rack gears were not too loose on the guide plate causing them to slip from the pinion. This was done by setting a tolerance in the dimension of each component that is half of the normal tolerance values.

\section{B. Autonomous Mode}

The drone uses a rack and pinion mechanism to carry the envelope, see Figure 16 for the detail. The retrieval mechanism was specifically designed to be lightweight and offer enough surface area to retrieve the envelope. The simple slim design allows for more space for the necessary electrical sensors and components. The device is located on the bottom of the micro vehicle. A 180-degree rotation mini servo is incorporated to provide a full range of motion for the pinion. The pinion is a cap that attaches to the rotor of the servo motor. The retrieval mechanism incorporates a sticky pad at the end of the rack to firmly pick up the envelope. The pad is sticky enough to hold the $25 \mathrm{~g}$ envelope, yet release the envelope for a smooth drop-off. Considering the high level of detail provided by the printer, the retrieval mechanism was 3D-printed on a FormLabs resin printer. The type of resin used is the tough variety offered by FormLabs to endure the cyclical process of pickup and delivery.

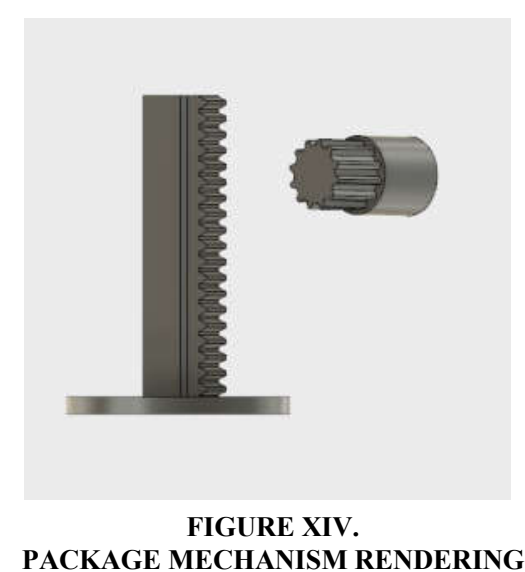

\section{Mathematical ANAlysis OF SySTem DeSIGN}

The CAD analysis of the frame and landing gear require the incorporation of various stresses. The stress of the designed object has a relationship with the force applied to the object and the area which the force applies. The expression is shown as follows.

$$
\sigma=\frac{F}{A}
$$

Where:

$$
\begin{aligned}
& \sigma=\text { Stress } \\
& \mathrm{F}=\text { Force } \\
& \mathrm{A}=\text { Cross-sectional area perpendicular to force applied. }
\end{aligned}
$$

Stress is the equivalent of a force distributed over an area. Strain is the ratio of deformation over the original length of the specimen. The equation for strain is as follows. 


$$
\varepsilon=\frac{\Delta L}{l}
$$

Where:

$$
\begin{aligned}
\varepsilon & =\text { Strain } \\
\Delta \mathrm{L} & =\text { Change in length of beam } \\
\mathrm{L} & =\text { Original length of beam }
\end{aligned}
$$

Each material has different properties that define the amount of stress and strain a material can withstand. The stress experienced on a part can be reduced into three principal stresses, one for the $\mathrm{x}, \mathrm{y}$, and $\mathrm{z}$ axis [\#]. The stress values are affected by the geometry of the part. The shearing stress the part endures from the normal stress affects how the part experiences the loading. Equation \#3 is the general equation for shear stress

$$
\tau=\frac{F}{A \prime}
$$

Where:

$$
\begin{aligned}
\tau & =\text { Stress } \\
\mathrm{F} & =\text { Force } \\
\mathrm{A} & =\text { Cross-sectional area parallel to force applied area. }
\end{aligned}
$$

Using Equations 1 and 3 the engineering concept of Mohr's circle can be applied. The Mohr's Circle is a graphical method of determining the maximum and minimum principal stress values.

Another indicator of part failure is the yield point of the material. The yield point of a material is derived from the physical and chemical properties of the material. Von Mises stress is used the analysis of the frame and landing gear [4] [5]. Von Mises stress determines when a material would yield by comparing the stress value to the material's maximum yield stress [2]. This process can be applied to multiple stresses applied to various planes. Equation 4 below expresses the use of Von Mises principles on the left-hand side of the expression.

$$
\left[\frac{\left(\sigma_{1}-\sigma_{2}\right)^{2}+\left(\sigma_{2}-\sigma_{3}\right)^{2}+\left(\sigma_{3}-\sigma_{1}\right)^{2}}{2}\right]^{1 / 2} \geq \sigma_{y}
$$

Where:

$\sigma_{\mathrm{i}}=$ Respective plane where the stress is located.

$\sigma_{\mathrm{y}}=$ Yielding Stress

\section{SySTEM WEIGHT ANALYSIS}

The frame design of the autonomous quadcopter, the total weight of the quadcopter in calculation came to be 460.7 grams. The main drone frame with all printed components, carbon fiber, and pickup mechanism. The weight of the final frame was slightly less than the manual frame due to the size requirements to mount all electrical components. From the table above it was seen that the heaviest electrical component onboard the micro air vehicle is the Odroid XU4 computer board because of the necessity of the heatsink and cooling fan. The Pixhawk flight controller when compared to the Flyduino weighs 8 grams more. This is due to the size and amount of ports available. Like the manual drone, the autonomous drone is powered by a Venom Fly 3S 1300 mAh LiPo battery which provides enough power to all onboard electronics to complete the competition tasks. In addition to the Venom Fly battery, the autonomous drone also came equipped with a X6R 16 channel receiver like the manual control quadcopter.

TABLE II.

AUTONOMOUS DRONE WEIGHT SUMMARY

\begin{tabular}{||c|c|c|c||}
\hline Item & Quantity & $\begin{array}{c}\text { Unit } \\
\text { Weight (g) }\end{array}$ & Total (g) \\
\hline Pixhawk Flight Controller & 1 & 19 & 19 \\
\hline Odroid XU4 Computer Board & 1 & 40 & 40 \\
\hline PX4 Optical Flow v1.3 & 1 & 19 & 19 \\
\hline $\begin{array}{c}\text { Lidar-Lite v3 Laser Ranging } \\
\text { Module }\end{array}$ & 1 & 20 & 20 \\
\hline Arris Brushless Motors & 4 & 18 & 72 \\
\hline Arris ESC & 4 & 5.3 & 21.2 \\
\hline Power Distribution Board & 1 & 5.5 & 5.5 \\
\hline X6R 16-channel Receiver & 1 & 15 & 15 \\
\hline Raspberry Pi Cam v2 & 1 & 2 & 2 \\
\hline Continuous Servo & 1 & 9 & 9 \\
\hline Venom Fly Lipo Battery & 1 & 108 & 108 \\
\hline Main Drone Frame & 1 & 110 & 110 \\
\hline Pickup/Drop-off Mechanism & 1 & 15 & 15 \\
\hline Miscellaneous Materials & 1 & 5 & 5 \\
\hline
\end{tabular}

The manual drone built was expected to be lighter in weight when compared to the autonomous drone. This is since there was no necessity for any sensors or computer boards such as the Odroid XU4 on the drone. Instead, the quadcopter will only require an onboard Mini-FPV camera to provide video feed to the pilot's FPV goggles [8]. The final total weight out the manual mode quadcopter was 407 grams. 
TABLE III. MANUAL DRONE WEIGHT SUMMARY

\begin{tabular}{||c|c|c|c||}
\hline Item & Quantity & $\begin{array}{c}\text { Unit } \\
\text { Weight (g) }\end{array}$ & Total (g) \\
\hline $\begin{array}{c}\text { Flyduino KISS CompactCTRL } \\
\text { FC }\end{array}$ & 1 & 11 & 11 \\
\hline Arris Brushless Motors & 4 & 18 & 72 \\
\hline 90 Degree Servo & 1 & 11 & 11 \\
\hline Continuous Servo & 1 & 11 & 11 \\
\hline Eyoyo Mini FPV Camera & 1 & 7 & 7 \\
\hline X6R 16-channel Receiver & 1 & 15 & 15 \\
\hline Venom FlyLipo Battery & 1 & 108 & 108 \\
\hline 5030 Propellers & 4 & 2 & 8 \\
\hline Main Drone Frame & 1 & 140 & 140 \\
\hline Pickup/Drop-off Mechanism & 1 & 100 & 100 \\
\hline Miscellaneous Materials & 1 & 5 & 5 \\
\hline \multicolumn{1}{|c|}{} & & Total: & $\mathbf{4 0 7}$ \\
\hline
\end{tabular}

\section{Autonomous Programming}

Achieving autonomous flight for Micro Aerial Vehicle is done with Robot Operating System (ROS) and a ROS package called MAVROS. The ROS framework is designed to work efficiently on Linux based computers and therefore it was decided to use Odroid XU4 -a single board computer- as the brain of this quadcopter and all the processing will be done on board. Basic ROS architecture is made up of nodes (publishers, subscribers), topics, services and messages. The MAVROS package allows the use of a protocol named MAVlink which consists of 17 bytes and includes the message ID, target ID, and data and allows the communication between the ground control station and unmanned vehicle. To send data, the user must "publish" the data to a specific topic. When the user would like to read or input data the user must "subscribe" to a topic. Topics are used to exchange messages across different systems. A service is like a topic that systems exchange messages but when a system uses a service to send a message that system waits for a reply before completing a new action. MAVROS handles all the communication between the flight controller unit (FCU) to ROS. After achieving successful communication user can subscribe and publish to all the MAVROS topics.

The MAVROS topic used to control the movement of the quadcopter is /setpoint_raw/local. This topic requires for the user to send $\mathrm{x}, \mathrm{y}$ and $\mathrm{z}$ coordinated in meters and yaw angle. The quadcopter is first armed with service /cmd/arming. The mode of the quadcopter is set to offboard using service /set_mode. After the quadcopter is set to offboard mode, the desired target position is sent.

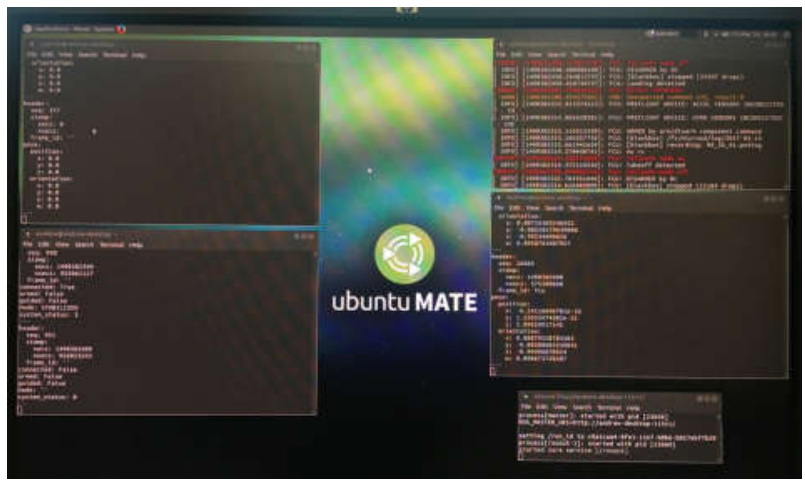

FIGURE XV.

MULTIPLE NODES RUNNING ON ODROID XU4

To extract the features from the ground to make the quadcopter stay at one place, a downward facing camera and LIDAR are used. To find different targets like the home base and pick up and drop off targets, different types of techniques are used. Currently the camera is connected to Odroid which is running a ROS node which is created using OpenCV. The OpenCV code uses a cascade classifier based on Haar-like features which are images used in object detecting algorithms. Haar features are used to reduce processing because computing images based on image intensity (Red, Green, Blue) can get processor intensive. The Haar-feature detector proposed by Paul Viola and Michael Jones classifies portions of images based on the adjacent bounding rectangles which are compared to a threshold to classify the portion on the image as positive (Object is present) or negative (Object is absent). For example, when classifying faces on an image, the eye section is different than the cheek section which can be used to generate a comparing result. Since, the classifier is based on comparing regions on a portion of image, the resulting classifier is weak.

To create a strong classifier, OpenCV utilizes the concept of boosted classifier. This algorithm takes weak classifiers and creates a strong classifier to identify objects successfully. To create an adequate target detector, a comprehensive data preparation was done. The cascade classifier was given 100 images of home base with 500 negative images. The positive images had to be marked up to identify where the home base was. The trained cascade results were incorporated in a $\mathrm{C}++$ code to draw bounding circles around a presumed portion of the frame captured by the on-board camera. The size of the bounding circles on the frame correspond to the cascade classifier results that the object is present proportioned to the image. The smaller the circle, the less of a chance for the home base to be present; the bigger the circle, the higher the chance of home base being present on in the frame. This code successfully identifies if the home basses is present in the frame.

After it has detected the $\mathrm{H}$, the $\mathrm{x}$, $\mathrm{y}$ coordinated are published to a user defined topic. In the script the controlling of the quadcopter is done with subscribed data. If the

$1^{\text {th }}$ LACCEI International Multi-Conference for Engineering, Education, and Technology: "Global Partnerships for Development and Engineering Education”, 19-21 July 2017, Boca Raton FL, United States. 
quadcopter doesn't get any $\mathrm{x}$, y values, then no home base was detected and it will continue to find $\mathrm{H}$. if $\mathrm{H}$ is detected, then hovering algorithm is used to hover on $\mathrm{H}$ for 5 seconds.

The hovering algorithm currently takes the found $\mathrm{H}$ and finds the displacement of the $\mathrm{H}$ from the middle of the frame (or middle of the quadcopter since the camera is mounted in the middle of the quadcopter) and the quadrant in which the $\mathrm{H}$ is in. Based on these displacements and the quadrant, slight correction values are sent to /setpoint_raw/local. Which results in stable hover over home base and similarly on pick up and drop off targets.

\section{ConClusion}

The objective of this project was to develop two micro air vehicles capable of completing the mission tasks set by the American Helicopter Society (AHS) International Micro Air Vehicle Competition 2017. One micro air vehicle was designed for manual operation while the other was designed for autonomous operation. Using the CATIA Computer Aided Design software, the two micro air vehicles were designed and stress analysis was performed to ensure that the frames will be able to withstand the thrust of the motors during flight and possible collisions. Both the autonomous and manual frame were designed consist of several different sections that could be replaced in the case of damage. The manual quad-copter uses a main cross shaped frame with a landing gear mechanism that can pick up and deliver the package. Due to the number of components onboard the autonomous quad-copter, the frame was designed using carbon fiber rods and PLA motor mounts and main body [9]. Both drones were designed in specific ways to dampen vibration from the motors, maintaining stability of the gyroscopes. In the final iterations of the design, it was seen through tasks that the both drones can achieve stable flight.

In the final competition, the manual controlled micro air vehicle completed the entire course winning $2^{\text {nd }}$ place in the manual category of the competition. Unfortunately, the autonomous drone was unable to complete the mission tasks even when stable flight and hover was achieved. Upon further inspections, it was discovered that the autonomous frame began vibrating and incapable of stable flight due to interference with the onboard electronics at the competition location.

\section{ACKNOWLEDGEMENTS}

The authors would like to thank Vaughn College of Aeronautics and Technology and the Department Chair of Engineering and Technology, Dr. Hossein Rahemi, for their enthusiastic efforts to consistently support the Unmanned Aerial Vehicles Club. Furthermore, we would like to thank the
UAV Club faculty advisor, Dr. Amir Elzawawy for his support and guidance.

\section{REFERENCES}

1 American Helicopter Society $5^{\text {th }}$ Annual MAV Student Challenge Final Rules, January 2017. Accessed January 2017.

2 http://www.learnengineering.org/2013/01/principal-stress-plane-mohrcircle.html, accessed by February 2017.

3 Hatfield, M.C., Monahan, J. and VanderWaal S., "UAS design with active learning," Proceeding of the $123^{\text {rd }}$ ASEE Annual Conference \& Exposition, New Orleans, June 2016, Paper ID \#15656. Accessed March 2017.

4 http://www.learnengineering.org/2012/12/what-is-von-mises-stress.html, accessed March 2017.

5 http://www.continuummechanics.org/vonmisesstress.html, accessed March 2017

6 http://www.dronetrest.com/t/power-distribution-boards-how-to-choosethe-right-one/1259, accessed by February 2017.

7 https://oscarliang.com/best-flight-controller-quad-hexcopter/, accessed by January 2017

8 http://www.droneflyers.com/what-is-this-fpv-stuff-all-about/, accessed by March 2017

9 https://www.memaster.com/\#standard-carbon-fiberhollowtubing/=172x3fk, accessed by April 2017.

10 http://www.popularmechanics.com/home/reviews/a8109whats-so-greatabout-brushless-motor-power-tools, Accessed by January 2017. 